\title{
Research on Behavior Model of Rumor Maker Based on System Dynamics
}

\author{
Xiaoqian Zhu and Fengming Liu \\ School of Management Science and Engineering, Shandong Normal University, Jinan 250014, China \\ Correspondence should be addressed to Fengming Liu; liufm@sdnu.edu.cn
}

Received 13 February 2017; Revised 7 May 2017; Accepted 16 May 2017; Published 21 June 2017

Academic Editor: Pasquale De Meo

Copyright (c) 2017 Xiaoqian Zhu and Fengming Liu. This is an open access article distributed under the Creative Commons Attribution License, which permits unrestricted use, distribution, and reproduction in any medium, provided the original work is properly cited.

\begin{abstract}
Laws of rumor makers' behaviors are the root of curbing rumor and effective way to block rumor occurrence. Therefore, based on system dynamics model, this paper proposed the rumors behavior evolution model of rumor makers, aimed at discovering the laws of rumor makers' behaviors to achieve rumors blocking. First, by refining the driving factors in disinformation behavior, we constructed causal diagram of disinformation behavior evolution; secondly, by means of causal diagram, we constructed stockflow diagram for quantitative analysis; finally, simulation experiment was carried out by using Vensim Personal Learning Edition software (Vensim PLE). The results showed that negative attitude is a major factor in the occurrence of disinformation behavior; personal factors are more pronounced than the factors of social and government on the impact of disinformation propensity score.
\end{abstract}

\section{Introduction}

As a kind of information, rumors can not only disturb people's daily life but also destroy economic development and social stability, and it must be strictly guarded and controlled. In addition to the spreaders which add fuel to the fire of rumors, there are lots of rumor makers who promote the spreading of rumors. Therefore, the study on behavior rules of rumor makers, which curbs the rumors at the root causes, becomes a hot spot for blocking the rumors.

At present, most of the research on behavior rules of rumor makers was conducted by means of statistical tools. Through a large number of historical data, they obtain the statistical characteristics of behavior rules and make a macroprediction of human behavior. Miritello [1] combs literature on statistics of human behavior in information dissemination and finds that the research method based on statistics of historical data is one of the effective methods to study the law of human behavior. Ma and others [2] confirmed that the tails of RT distributions exhibit power law behavior. Therefore, through collecting and analyzing broadcast data sent by 140 Twitter users, Salathé [3] and others based on the theory of psychology conclude that the emotions from friends and social awareness are highly correlated with individual information production behavior; that is, the emotions from neighbors have an important impact on information manufacturing behavior. Based on hierarchical temporal memory, Li [4] and others construct a cognitive model of rumors makers. They simulate the cognitive process of rumors in heterogeneous groups with different knowledge and personal experience and conclude that the manufacturing of rumor is driven by social cognitive factors. Moreover, system dynamics are a powerful tool for studying causality and can be used to analyze driving factors. Based on a system dynamics approach and the net anthropogenic $\mathrm{N}$ input (NANI) concept, a NANI-SD model [5] was developed to simulate the relationship between NANI and its drivers. Then, the system dynamics model developed in this study identified key factors influencing regional anthropogenic $\mathrm{N}$ input. Therefore, in order to discover rumor maker's behavior law, we will find behavioral driving factors of rumor maker and construct dynamics mode of disinformation behavior. It is an important method in the field of net rumors. This paper combs the related literature in the field of network rumor and excavates the behavioral drivers of rumor maker. Based on system dynamics theory, this paper puts forward 
TABLE 1: Rumor-driven factors.

\begin{tabular}{lc}
\hline Factor categories & Included factors \\
\hline Personal factors (PF) & Event attention (EA), personal discernment (PD), negative mentality (NM) \\
Social factors (SF) & Group polarization (GP), social trust (ST), mass discussion frequency (DF) \\
Governmental factors (GF) & Governmental regulation (GR), political activity (PA), dissemination efforts (DE) \\
\hline
\end{tabular}

the disinformation behavior evolution model of the rumor maker. The rumor maker's behavior evolution is a dynamic process influenced by many factors. This paper analyzes these factors from the personal factors, the social factors, and the governmental factors. In this paper, we use rumor tendency (RT) as a quantitative index to reflect the impact on information contacts. The higher the RT is, the higher the probability that the information contacts become rumor makers will be. And, the lower the RT is, the less likely that the information contacts become rumor makers will be.

\section{Related Work}

In the field of rumors driver, many scholars have conducted research and achieved a series of results; most of the research focused on the field of human psychology [6]. As early as 1945, American personality psychologists Allport and Postman suggested that any human demand can provide rumor for power [7]. Later, Difonzo and Bordia [8] considered that rumor transmission is motivated by three broad psychological motivations: fact-finding, relationship enhancement, and self-enhancement. Rumor is closely entwined with a host of social and organizational phenomena, including social cognition, attitude formation and maintenance, prejudice and stereotyping, group dynamics, interpersonal and intergroup relations, social influence, and organizational trust and communication. Through experiments, Ajzen and Fisbbein [9] confirmed the conclusion that behavioral intentions correlated significantly with behavior. During emergency events, individuals are exposed to large quantities of information without being aware of their validity or risk of misinformation, but users are usually swift to correct them, thus making the social media "self-regulating" [10]. Insofar as some people's behavior is controlled by custom and convention, it is a product of society, of the individual's interpretation of his role, and so, indirectly, of collective action [11].

The impact of the social environment on RT is also crucial. Through analytic derivation and simulations, Shaw and others [12] found that gossip destroys clustering in weakly clustered networks and increases cliquishness in networks with already high clustering. Hu and others [13] suggested that lower interpersonal influence of weak ties increases the isolation of social groups; thus, collectivism is unfavorable to the spread of participation across whole network, and they also demonstrated the importance of national culture on collective action. On the basis of previous studies, this paper will be discuss rumors-driven factors divided into three categories, as shown in Table 1.

\section{System Dynamics Simulation Model of Rumor Behavior Evolution}

In the micronetworks (WeChat, microblogging, etc.), all individuals must become information producers but not necessarily rumor makers. Social, psychological, personal, and other internal and external environmental factors could lead to the transformation of the information maker to the rumor maker; the impact of different factors is different. Therefore, mining driving factors and studying the extent of its impact, we could grasp the evolution laws of rumors, to achieve rumors blocking.

System dynamics are a useful tool for studying the causal relationship between factors. By analyzing the causal relationship between factors in the dynamic process of behavior evolution, the causal relationship diagram is constructed and the system dynamics modeling is implemented. Causal relationship diagram is a one-way complex network diagram composed of the influencing factors and the causal relationship among these factors, where the factor represents the node of the network; if there is a causal relationship between any two factors, there is a one-way edge between the two points that points to the result node by the cause node. If the result node changes in the same direction as the reason node, there is a positive causal chain between the two nodes. Otherwise it is called negative causal chain. When the causal chain is the same at the beginning and end, it forms a causal loop. At the same time, the polarity of the causal loop is determined by the number of positive and negative causal relationships in the loop.

3.1. Causal Analysis of the Disinformation Behavior Drivers. In the micronetworks, the behavior-driven factors of rumor makers are mainly composed by the personal factors, the social factors, and the governmental factors.

3.1.1. Personal Factors. The personal factors are determined by the heterogeneity of the individual in the network [3], which refers to the specific attributes of the individual, including the event attention, the personal discernment, and the negative mentality.

Event attention depends on age, occupation, education, the region's network coverage, and other inherent attributes of the individual. The personal discernment refers to the information judgment ability, the information processing ability, and the ability to overcome position bias for individual, and these abilities are restricted to individual learning, cognition, and experience. Negative mentality refers to the influence of network rumor psychological causes. Therefore, personal discernment and negative mentality are the direct factors of 


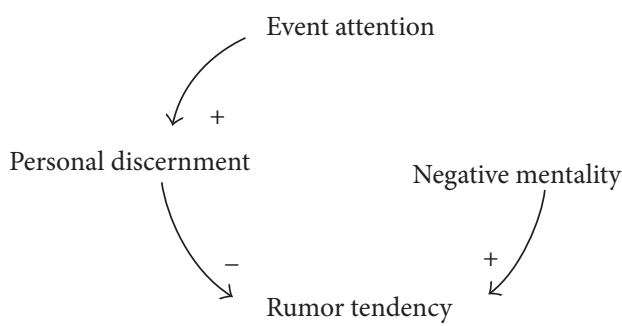

FIgURE 1: The personal factors causality diagram.

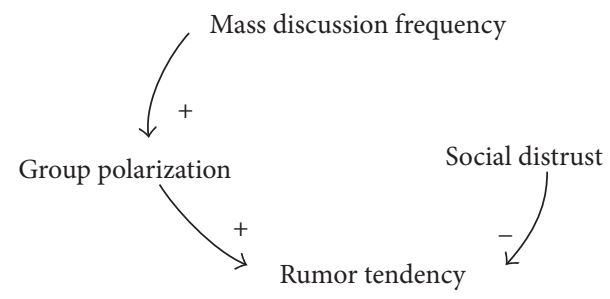

FIGURE 2: The social factors causal diagram.

$\mathrm{RT}$, while event attention is indirectly affected by personal discernment.

The causality diagram of personal factors is shown in Figure 1.

3.1.2. Social Factors. Social factors refer to influencing factors from the surrounding groups, organizations, or media, including the group polarization, the social trust, and the mass discussion frequency.

Group polarization means that biased ideas of individual will produce more extreme negative effects when receiving the opinion of the group. Mass discussion frequency refers to the proportion of communication behavior between individual nodes, which will directly affect the influence scope and influence degree of group polarization. Social trust refers to the score of social trust, and low trust between members of the community easily leads to a crisis of confidence. In social factors, the social trust and the group polarization have a direct influence on RT.

Social factors causal diagram is shown in Figure 2.

3.1.3. Governmental Factors (GF). Governmental factors refer to the impact of government taking regulatory actions, including the governmental regulation, the political activity, and the dissemination efforts.

As a regulator of networks, the government is a balancing mechanism for disinformation behavior. Government regulation refers to the government's enforcement of existing laws and regulations, as well as the emergency mechanism for future events. Political activity refers to the degree of expression and participation in political affairs. Dissemination efforts refer to the objective expression of the opinions and the subjective acceptance of the audience. Political activity and dissemination efforts are the two-major government index. The high RT promotes the political activity,

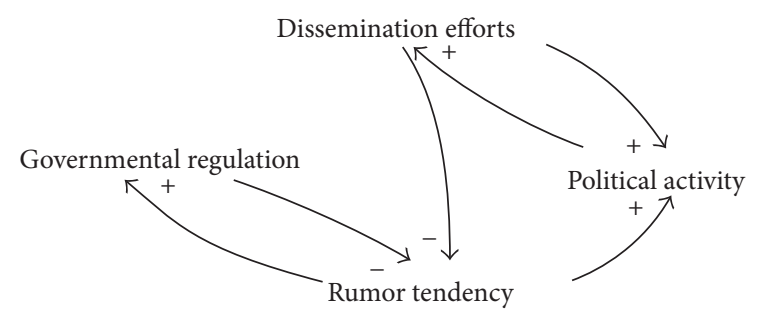

Figure 3: The governmental factors causal diagram.

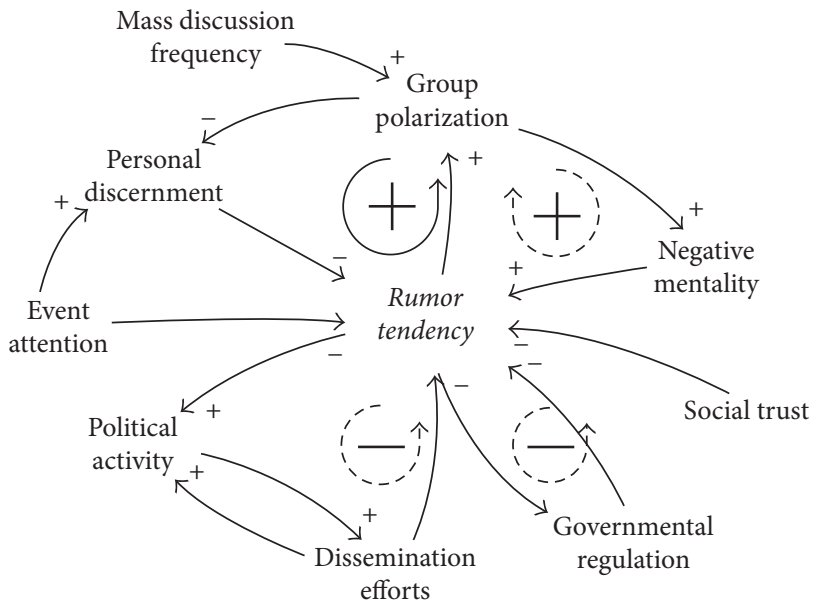

FIGURE 4: The disinformation behavior drivers causal-loop diagram.

strengthens the dissemination efforts, and thus reduces the RT. Finally, the balance of RT will be realized.

Figure 3 shows the governmental factor causality diagram.

3.1.4. Causal-Loop Diagram of the Disinformation Behavior Drivers. Figure 4 shows the causal-loop diagram of the disinformation behavior drivers. There are two positivefeedback loops and two negative-feedback loops.

(1) "RT" $\rightarrow$ "group polarization" $\rightarrow$ "personal discernment" $\rightarrow$ "RT" (positive-feedback loop 1)

(2) "RT" $\rightarrow$ "group polarization" $\rightarrow$ "negative mentality" $\rightarrow$ "RT" (positive-feedback loop 2)

Two positive-feedback loops indicate that RT is directly driven by two factors: the personal discernment and the negative mentality. Considering personal factors, the group polarization is indirect to the RT. Through the group polarization, RT also could weaken the personal discernment and contribute to negative mentality.

(3) "RT" $\rightarrow$ "political activity" $\rightarrow$ "dissemination efforts" $\rightarrow$ "RT" (negative-feedback loop 1)

(4) "RT" $\rightarrow$ "governmental regulation" $\rightarrow$ "RT" (negativefeedback loop 2)

As immature control techniques and unsound laws and regulations, the government often takes ex post measures of the management of network rumors. Only when rumors 


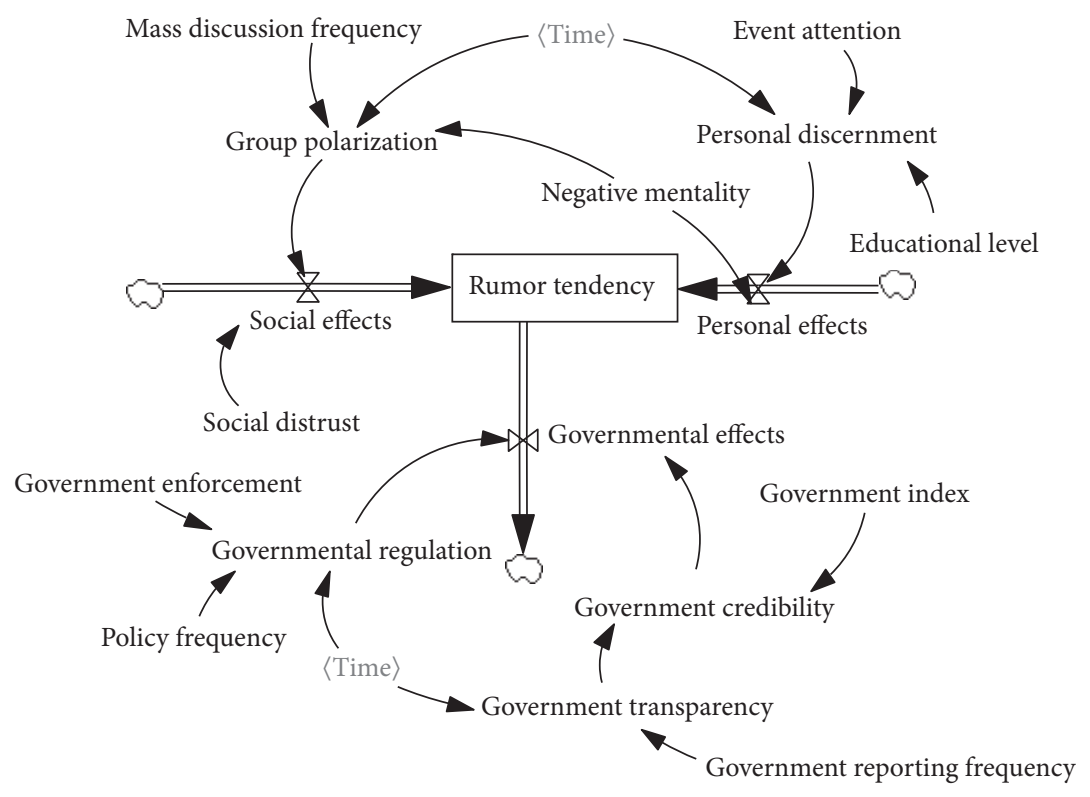

FIGURE 5: Flow-stock diagram of the disinformation behavior drivers.

endanger economic security and social stability will the government come forward to dispel rumors and strengthen supervision. The governmental regulation is not enough, and the RT will increase. In the society with high RT, strengthening the governmental regulation is helpful to safeguard government image and improve the credibility of the government, thus blocking the upward spiral of RT. The negative-feedback loop between the RT and the governmental regulation constitutes an important balance relationship. Therefore, government influence is a balance mechanism of disinformation behavior.

3.2. Flow-Stock Diagram of the Disinformation Behavior Drivers. The flow-stock diagram is another effective tool for system dynamics modeling. The causality diagram could qualitatively reflect the feedback structure of the system, that is, the causal relationship among the driving factors. Giving the variable dynamic accumulation, the flow-stock diagram is quantitative analysis of the system. In the stockflow diagram, the stock in the feedback system represents the accumulation of the stock. The flow is represented by the change in stock over time, and the difference between the inflow and outflows produces the stock. In this paper, the RT is defined as the stock of disinformation behavior evolution model, and it is used to characterize the rumor state. Simultaneously, personal effects, social effects, and governmental effects are defined as the flow; these factors work together to influence the RT. The flow-stock diagram of the disinformation behavior drivers is shown in Figure 5.

\subsubsection{Variable Formulas of the Disinformation} Behavior Drivers

(1) Personal discernment $=$ event attention (time) $*$ educational level
(2) Personal effects $=\mathbf{1} /\{(\mathbf{1}-$ negative mentality $) *$ personal discernment

(3) Government transparency = government reporting frequency (time)

(4) Government credibility $=a *$ government index + $b *$ government transparency

(5) Governmental regulation = policy frequency $($ time $)+$ government enforcement

(6) Governmental effects $=c *$ government credibility + $d *$ governmental regulation

(7) Group polarization = mass discussion frequency (time) $/(1$ - negative mentality)

(8) Social effects $=$ social distrust $*$ group polarization

(9) Rumor tendency $=$ integer $(\alpha *$ social effects + $\beta *$ personal effects $-\theta *$ governmental effects)

20 experts and sociologists which from the field of net rumors graded the importance of each factor, and we got the following conclusions. The weights of government index and government transparency are 0.3 and 0.7 , respectively. And, government credibility and governmental regulation are obtained by the expert scoring method as, respectively, 0.7 and 0.3 . The weight of social effects, personal effects, and governmental effects are $0.164,0.583$, and 0.253 .

\section{Simulation and Analysis of Model}

4.1. Simulation of Disinformation Behavior Evolution Model. Based on the public opinion case statistics of China public sentiment network, this paper defines and initializes the model variables by means of table functions and expert scoring method. The event attention, the government reporting frequency, and the mass discussion frequency indicate, 


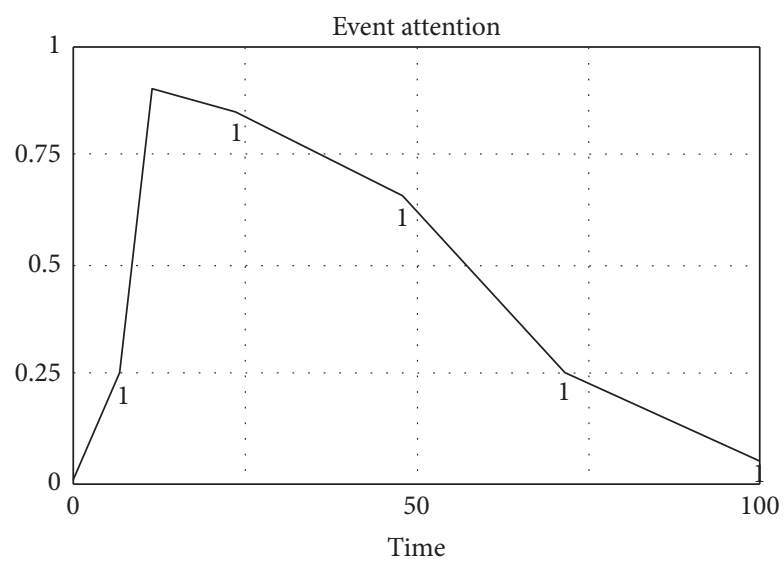

1 Initial state

(a)

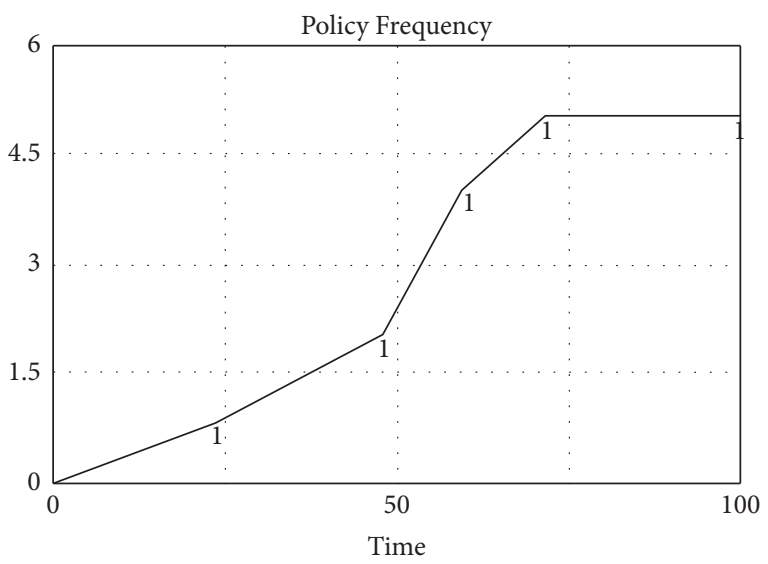

1 Initial state

(c)

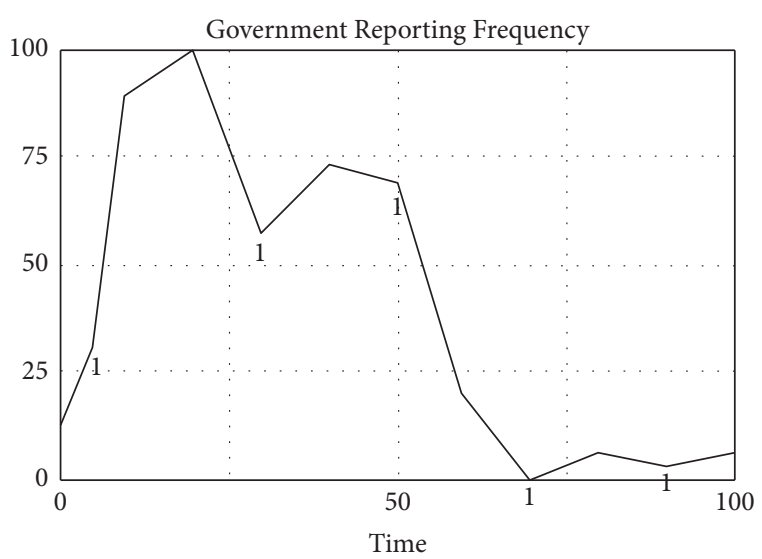

1 Initial state

(b)

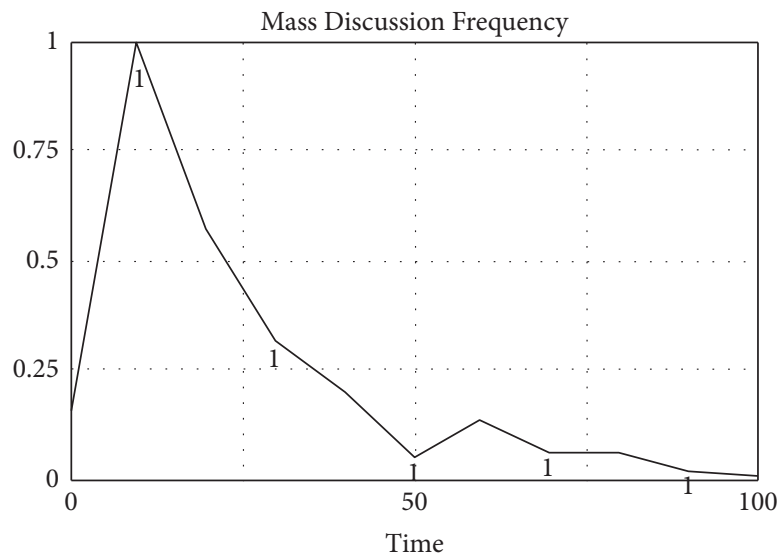

1 Initial state

(d)

FIGURE 6: Table function representation of each variable (event attention shown in (a); government reporting frequency shown in (b); policy frequency shown in (c); mass discussion shown in (d)).

TABLE 2: Table function of event attention.

\begin{tabular}{lccccccc}
\hline Time (hour) & 0 & 7 & 12 & 24 & 48 & 72 & 100 \\
\hline Event attention frequency & 0.01 & 0.25 & 0.9 & 0.85 & 0.65 & 0.25 & 0.05 \\
\hline
\end{tabular}

TABLE 3: Table function of government reporting frequency.

\begin{tabular}{|c|c|c|c|c|c|c|c|c|c|c|c|c|}
\hline Time (hour) & 0 & 5 & 10 & 20 & 30 & 40 & 50 & 60 & 70 & 80 & 90 & 100 \\
\hline Government reporting frequency & 12 & 30 & 89 & 100 & 57 & 73 & 69 & 20 & 0 & 6 & 3 & 6 \\
\hline
\end{tabular}

respectively, the attention of the individual, the government, and the society to the hot issues (Figure 6). With the dynamic evolution, these variables often show irregular distribution. Therefore, this paper uses the table function method to express the above variables, as shown in Tables 2-5.

In the model, the government index, the government enforcement, the educational level, the negative mentality, and the social distrust are all constants. According to the expert scoring method and optimization of the simulation results, these constants' initial values are $80,75,45,0.5$, and 25, respectively, while the experimental results are more intuitive.

\subsection{Analysis of Model Simulation Results}

4.2.1. Rationality Analysis. According to the setting of the model parameters and the establishment of the variable formula, the simulation diagram of the RT is obtained. As can be seen from Figure 7, we know that the RT is to change dynamically over time. In the early stage of disinformation 
TABLE 4: Table function of policy frequency.

\begin{tabular}{lcccccc}
\hline Time (hour) & 0 & 24 & 48 & 60 & 72 & 100 \\
\hline Policy frequency & 12 & 30 & 89 & 100 & 57 & \\
\hline
\end{tabular}

TABLE 5: Table function of mass discussion frequency.

\begin{tabular}{lccccccccccc}
\hline Time (hour) & 0 & 10 & 20 & 30 & 40 & 50 & 60 & 70 & 80 & 90 & 100 \\
\hline Mass discussion frequency & 0.157 & 0.997 & 0.57 & 0.312 & 0.194 & 0.045 & 0.134 & 0.054 & 0.06 & 0.017 & 0.01 \\
\hline
\end{tabular}

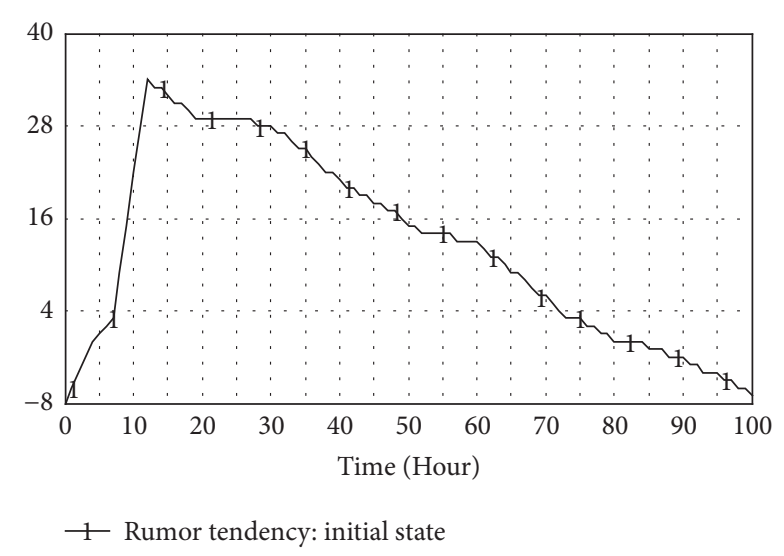

FIgURE 7: The rumor tendency graphs.

behavior, the RT will increase significantly. When reaching the peak, it begins to decrease slowly and finally returns to the initial state.

Because of the parametric hypothesis, the simulation results could not be completely consistent with the actual results. However, in the early stage of disinformation behavior, people will question or even contradict it. At this point, the RT is negative. With the increasing attention to the incident, RT is rising rapidly. When the rumors have finished disinformation, their attention to the incident will slowly reduce. And ultimately, they lose interest and unsubscribe it. It is concluded that the disinformation behavior evolution model is reasonable and could be used to simulate the disinformation behavior.

4.2.2. Sensitivity Analysis. Sensitivity analysis is that we could observe the changes of amount of dependent variable by changing the specific variables. And we could analyze the effect of variables on the dependent variable. By, respectively, changing the government index, the government enforcement, the educational level, the negative mentality, and the social distrust, we could observe the change of the RT and find out the main cause of disinformation behavior.

4.2.3. Personal Factors. In personal factors, the educational level (curve 2) and the negative mentality (curve 3) were increased by $30 \%$. The change of the RT is shown in Figure $8(\mathrm{a})$. The influence of negative mentality on RT significantly is higher than the education level; that is, an information contact with negative mentality is more likely to become a rumor maker. And, the Indian psychologist Beside has also raised the fact that the unrest is one of the motivations of rumors [7]. At the same time, when raising the educational level, RT tends to increase. This indicates that the higher the educational level, the higher the possibility of rumors. Figure 8(b) shows the effect of personal factors on the personal effects. (Curve 1 shows the initial state dynamic changes of disinformation tendency and is used to compare the changes in each factor.)

4.2.4. Social Factors. In social factors, this paper observes the impact of social factors on the RT, by increasing the initial state of social distrust (curve 2) by 30\%, as shown in Figure 9(a). The influence of social distrust on the RT is more significant; that is, social distrust will promote rumors. However, the social trust and the social distrust are reversechanged. Therefore, improving social trust can effectively control the rumors. Figure 9(b) shows the impact of social distrust on the social effects. From curve 2, we can see that the effect of social distrust on the rumor has a significant impact in the initial period. At this point, misleading information from the social environment and surrounding friends can directly affect behavioral choices of information contactors. And the contactor is most likely to become a rumor maker in the early stages.

4.2.5. Governmental Factors. In government factor, this paper reduces government enforcement (curve 2) and government index (curve 3 ) by $30 \%$, as shown in Figure 10(a). Government is a balancing act of rumors and should have commensurate influence and control. However, the enforcement and government index on the role of RT are not significant. At present, the real-name operations cannot be covered in the whole network, and human beings whose the real identity is not informed are prone to crime. That is to say, the anonymity of the networking and rumors of sudden and other characteristics lead to more complex network environment. The government often takes action after the rumor broke out, which causes government supervision and management on the network is difficult to achieve, especially for the disinformation behavior. Due to the "hysteresis" of the punishments, the deterrent effect of the government on the rumors is ignored. In Figure 10(b), with the same magnitude of decline, the enforcement for government impact is more significant. At the same time, unlike personal factors and social factors, the impact of government has effects throughout the whole rumor behavior evolution process. 

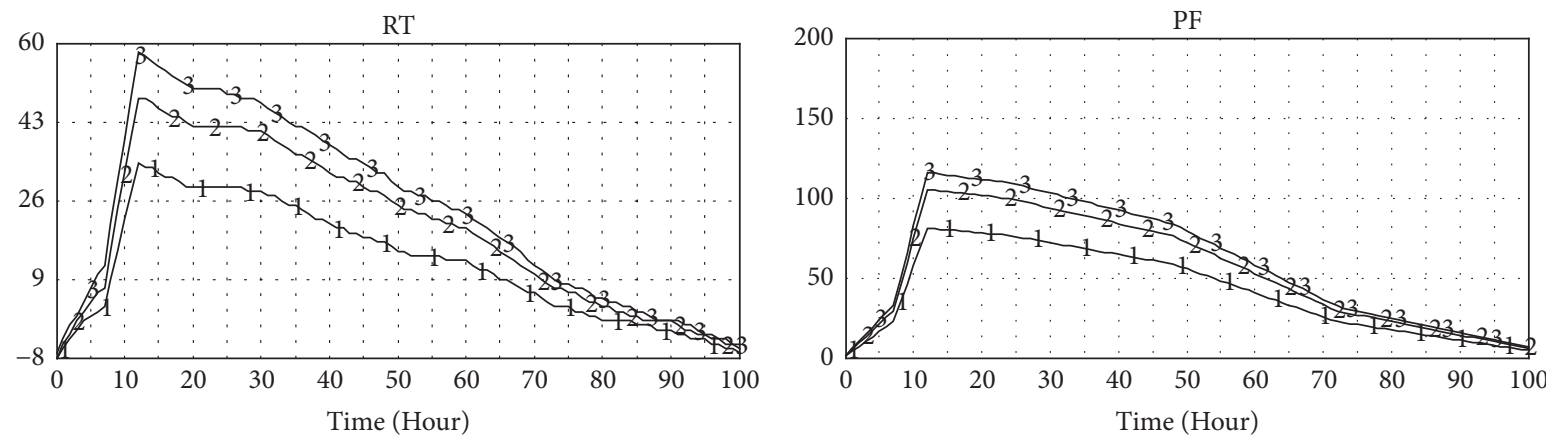

1 RT: initial state

2 RT: $30 \%$ increase in education level

3 RT: $30 \%$ increase in negative mentality

(a) The effect of personal factors on the rumor tendency

1 PF: initial state

2 PF: $30 \%$ increase in education level

3 PF: $30 \%$ increase in negative mentality

(b) The effect of personal factors on the personal effects

Figure 8
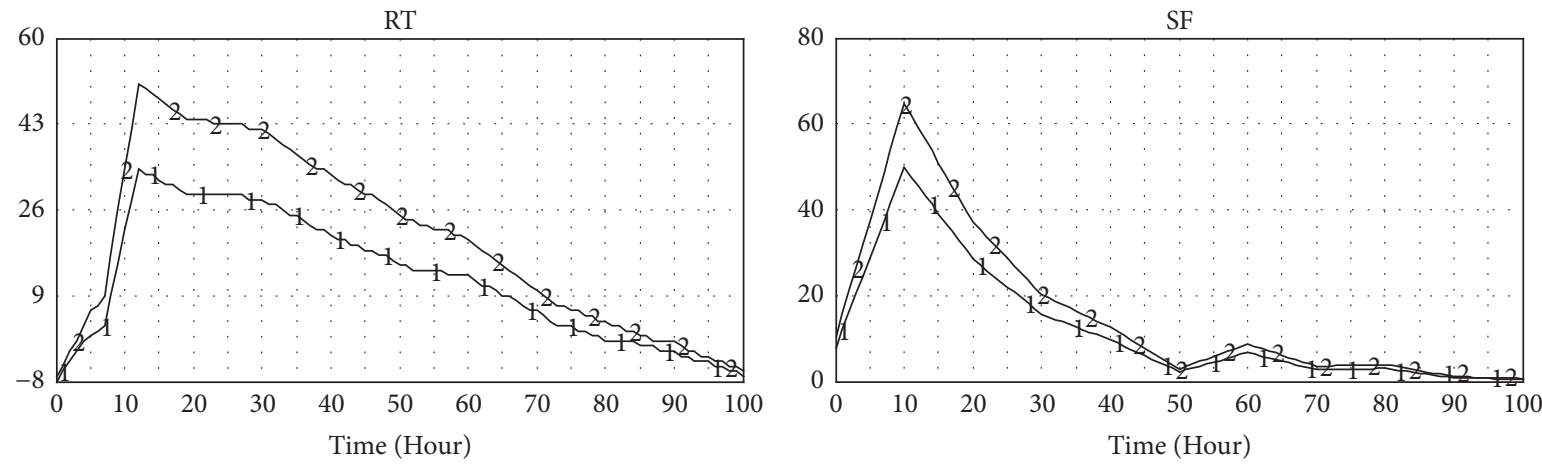

1 RT: initial state

2 RT: $30 \%$ increase in social distrust

1 SF: initial state

2 SF: $30 \%$ increase in social distrust

(a) The effect of social factors on rumor tendency

(b) The effect of social factors on the social effects

Figure 9
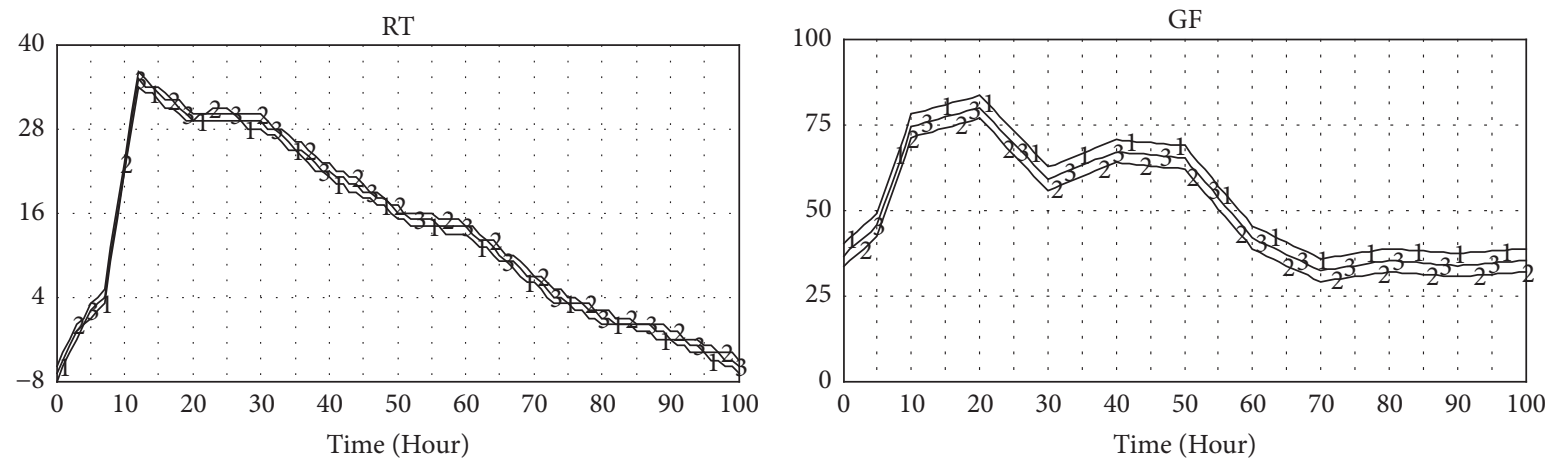

1 RT: initial state

2 RT: $30 \%$ reduction in government enforcement

3- RT: $30 \%$ reduction in government index

1 GF: initial state

2 GF: $30 \%$ reduction in government enforcement 3- GF: $30 \%$ reduction in government index

(a) The effect of governmental factors on rumor tendency

(b) The effect of governmental factors on governmental effects 


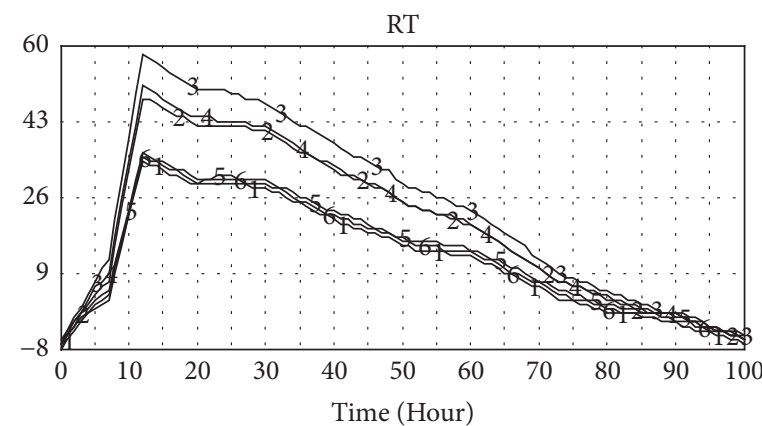

$$
\begin{array}{llll}
1- & \text { RT: initial state } & -4 & \text { RT: } 30 \% \text { increase } \\
2- & \text { RT: } 30 \% \text { increase } & \text { in social distrust } \\
\text { in education level } & -5 & \text { RT: } 30 \% \text { reduction in } \\
3-\text { RT: } 30 \% \text { increase } & \text { government enforcement } \\
\text { in negative mentality } & -6 & \text { RT: } 30 \% \text { reduction in } \\
& & \text { government index }
\end{array}
$$

(a) Analysis on the driving factors

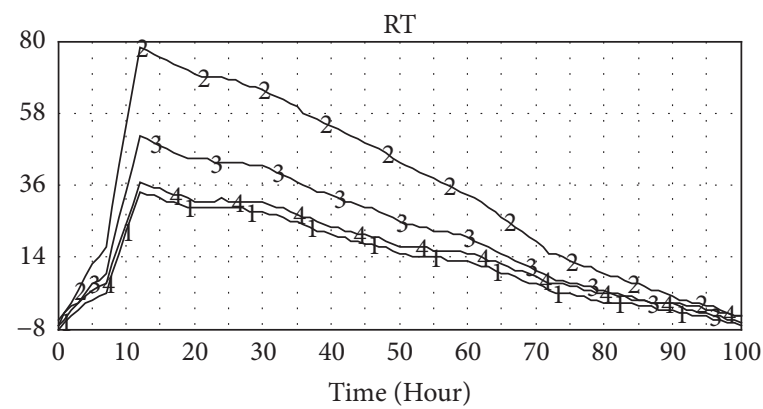

1 RT: initial state $\quad 3-$ RT: social factors

2 RT: personal factors $\quad-4$ RT: governmental factors

(b) The influence of various subsystems on rumor tendency

FIGURE 11

4.2.6. Comprehensive Factor Analysis. In Figure 11(a), we combine the various factors of three models. Under the same amplitude changes, the influence of negative mentality on the RT is the most significant. Changes in negative mentality are most sensitive to rumors. And negative mentality is the main motivation in promoting the disinformation behavior. As shown in Figure 11(b), the effect of personal effects on RT is the most significant, followed by social effects. The government effects have only a subtle effect on the rumor tendency. Therefore, personal factors are the key factors of disinformation behavior.

\section{Conclusions}

Network rumors endanger national security and social stability. The traditional network rumor propagation model aims to achieve blocking and governance of rumors. Their object is existing and destructive network rumors. However, by the system dynamics, this paper puts forward disinformation behavior evolution model of the rumor maker. This mode solves network rumors from the origin and provides a basis for monitoring and early warning of network rumors. The model is simulated from three aspects, individual, society, and government, and draws the following conclusions.

The influence of personal factors on RT is the most significant. Negative mentality is the main cause of disinformation behavior. The more the negative mentality of information contacts, the greater the possibility of disinformation behavior. Therefore, improving people's better life index and reducing social instability are conducive to reducing the negative mentality of the masses and controlling rumors. For the government, the key to reducing the RT is the government's control efforts and measures introduced efficiency. Strengthen the rumor punishment mechanism; improve the emergency response to emergencies. Through the "micro" platform, E-government could achieve network guidance, mass interaction, and so forth and, then, could standardize the network environment.

\section{Conflicts of Interest}

The authors declare no competing financial interests.

\section{Authors' Contributions}

Zhu Xiao-Qian and Liu Feng-Ming wrote the main manuscript text and Zhu Xiao-Qian prepared the experiments. All authors reviewed the manuscript.

\section{Acknowledgments}

This work was supported in part by the National Natural Science Foundation of China (no. 61170038, 61472231), the National Social Science Foundation of China (no. 14BTQ049), and a Project of International Cooperation in Training of Excellent Backbone Teachers for Advanced University in Shandong Province.

\section{References}

[1] G. Miritello, Temporal Patterns of Communication in Social Networks, Springer International Publishing, 2013.

[2] T. Ma, J. G. John, and R. A. Holden, "Distribution of human response times," Complexity, vol. 21, no. 6, pp. 61-69, 2016.

[3] M. Salathé, D. Q. Vu, S. Khandelwal, and D. R. Hunter, "The dynamics of health behavior sentiments on a large online social network," EPJ Data Science, vol. 2, no. 1, pp. 1-12, 2013.

[4] X. Li, X. Chen, and W. Wang, "Life system modeling and simulation: international conference on life system modeling and simulation, Lsms," in Proceedings of the International Conference on Intelligent Computing for Sustainable Energy and Environment (Icsee '14), pp. 268-277, Springer Berlin Heidelberg, Shanghai, China, September 2014. 
[5] W. Gao, B. Hong, D. P. Swaney, R. W. Howarth, and H. Guo, "A system dynamics model for managing regional $\mathrm{N}$ inputs from human activities," Ecological Modelling, vol. 322, pp. 82-91, 2016.

[6] P. E. Smaldino and J. C. Schank, "Human mate choice is a complex system," Complexity, vol. 17, no. 5, pp. 11-22, 2012.

[7] G. W. Allport and L. J. Postman, "Section of psychology: the basic psychology of rumor," Transactions of the New York Academy of Sciences, vol. 8, pp. 61-81, 1945.

[8] N. Difonzo and P. Bordia, Rumor Psychology: Social and Organizational Approaches, American Psychological Association, Washington, DC, USA, 2007.

[9] I. Ajzen and M. Fisbbein, "Factors Influencing Intentions and the Intention-Behavior Relation," Human Relations, vol. 27, no. 1, pp. 1-15, 1974.

[10] T. Simon, A. Goldberg, and B. Adini, "Socializing in emergencies-a review of the use of social media in emergency situations," International Journal of Information Management, vol. 35, no. 5, pp. 609-619, 2015.

[11] R. E. Park, "Human nature and collective behavior," American Journal of Sociology, vol. 32, no. 5, pp. 733-741, 1927.

[12] A. K. Shaw, M. Tsvetkova, and R. Daneshvar, "The effect of gossip on social networks," Complexity, vol. 16, no. 4, pp. 39-47, 2011.

[13] H.-H. Hu, J. Lin, and W. Cui, "Cultural differences and collective action: a social network perspective," Complexity, vol. 20, no. 4, pp. 68-77, 2015. 


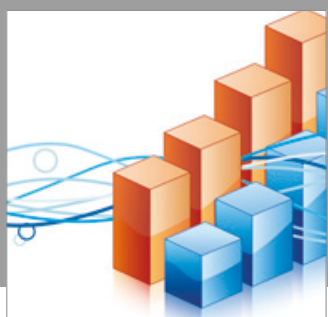

Advances in

Operations Research

vatersals

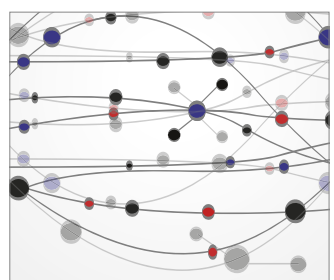

\section{The Scientific} World Journal
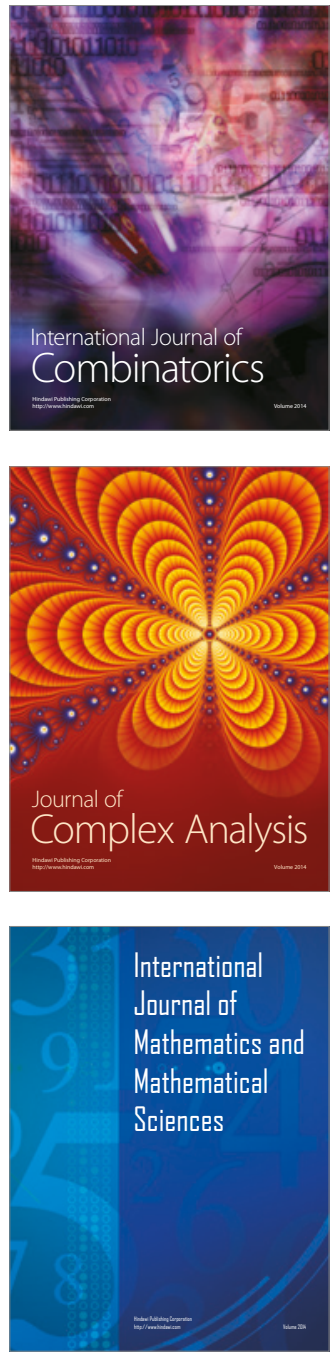
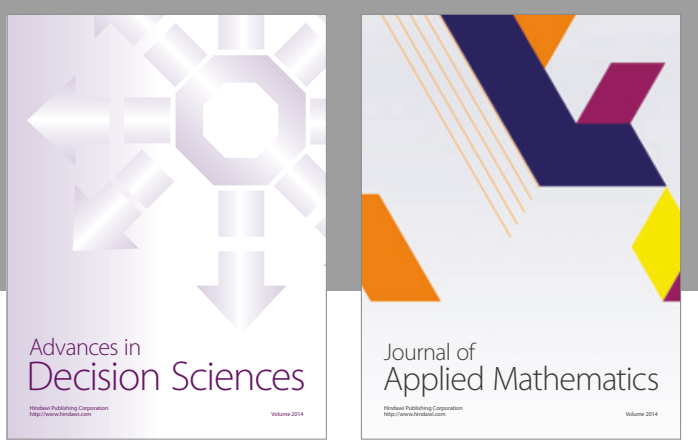

Algebra

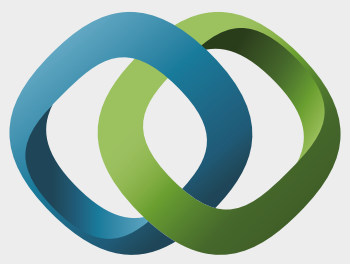

\section{Hindawi}

Submit your manuscripts at

https://www.hindawi.com
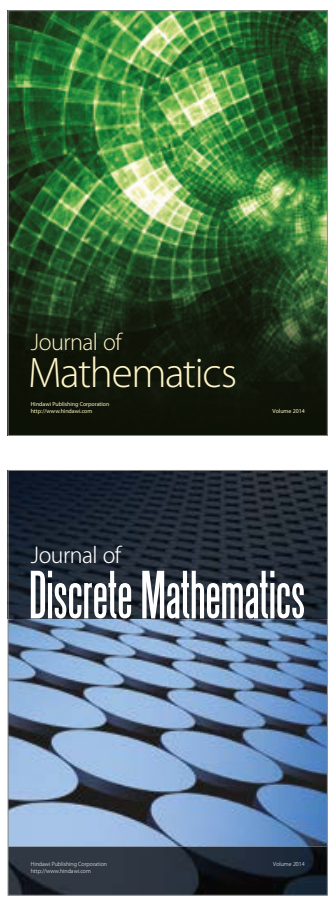

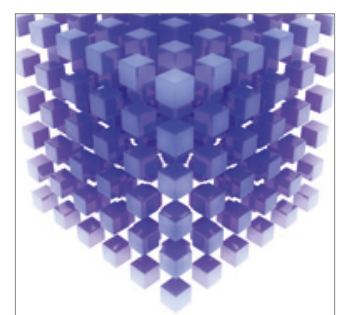

Mathematical Problems in Engineering
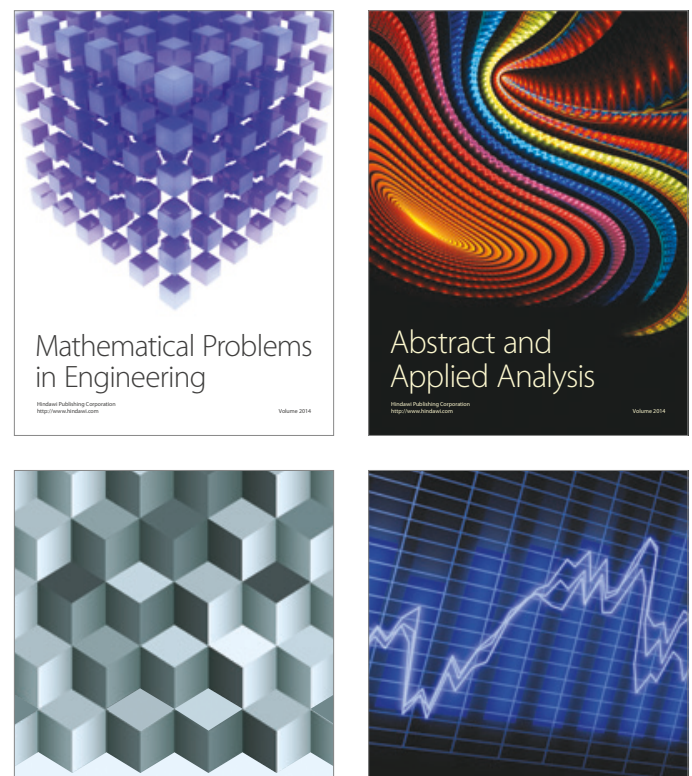

Journal of

Function Spaces

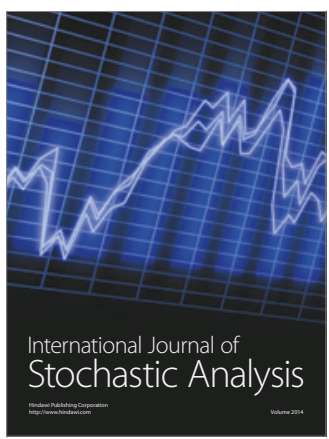

Probability and Statistics
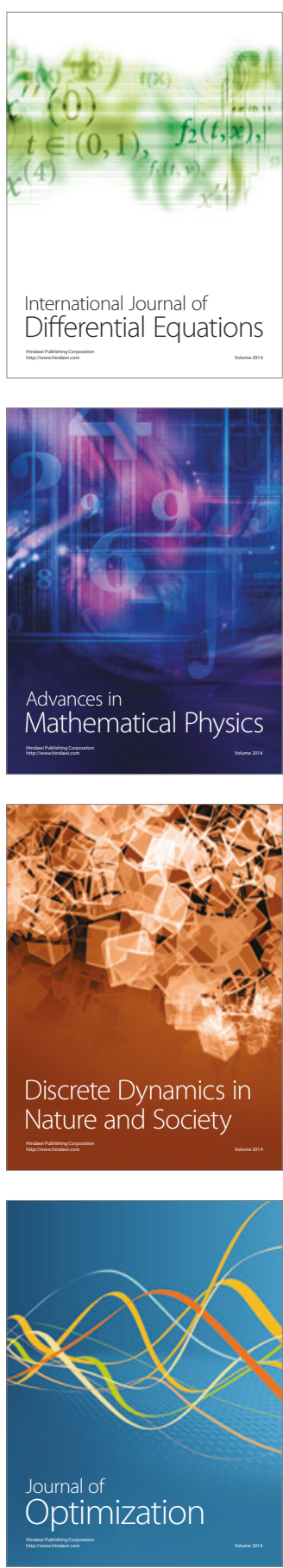